Tropical Journal of Pharmaceutical Research November 2021; 20 (11): 2255-2260

ISSN: $1596-5996$ (print); 1596-9827 (electronic) (C) Pharmacotherapy Group, Faculty of Pharmacy, University of Benin, Benin City, 300001 Nigeria

\title{
CircZNF652 accelerates the proliferation and migration of primary lung carcinoma cells by downregulating miR-766
}

\author{
Xiaobing Hong*, Yongqing Chen, Danzhen Zhang \\ Department of Respiratory and Critical Care Medicine, Taizhou Hospital of Traditional Chinese Medicine, Taizhou, China
}

*For correspondence: Email: 2329418023@qq.com; Tel: +86-0576-88825615

Sent for review: 9 August 2021

Revised accepted: 2 October 2021

\begin{abstract}
Purpose: To explore the biological functions and molecular mechanism of circZNF652 involvement in primary lung carcinoma.

Methods: CircZNF652 levels in primary lung carcinoma cases and controls were determined using quantitative real-time polymerase chain reaction (qRT-PCR). Its prognostic value in primary lung carcinoma was examined by depicting it with Kaplan-Meier curves. The biological functions of circZNF652 in regulating proliferative and migratory capacities in A549 and SPC-A-1 cells were analyzed from the curves. Interaction between circZNF652 and its downstream gene, miR-766, was assessed, and their co-regulation on primary lung carcinoma was determined by rescue experiments. Results: CircZNF652 was abnormally and significantly upregulated in primary lung carcinoma cases ( $p$ $<0.05)$, resulting in a poor prognosis. The knockdown effect of circZNF652 attenuated the proliferative and migratory capacities of A549 and SPC-A-1 cells, and downregulated epithelial-mesenchymal transition (EMT)-associated genes. CircZNF652 bound and negatively regulated miR-766, a key downstream gene involved in circZNF652-induced aggravation of primary lung carcinoma.

Conclusion: CircZNF652 serves as an oncogene, triggering the aggravation of primary lung carcinoma by negatively regulating miR-766. The results of this study may provide new insights into the treatment of lung carcinoma.
\end{abstract}

Keywords: CircZNF652, Primary lung carcinoma, MiR-766, Epithelial-mesenchymal transition

\begin{abstract}
This is an Open Access article that uses a funding model which does not charge readers or their institutions for access and distributed under the terms of the Creative Commons Attribution License (http://creativecommons.org/licenses/by/4.0) and the Budapest Open Access Initiative (http://www.budapestopenaccessinitiative.org/read), which permit unrestricted use, distribution, and reproduction in any medium, provided the original work is properly credited.
\end{abstract}

Tropical Journal of Pharmaceutical Research is indexed by Science Citation Index (SciSearch), Scopus, International Pharmaceutical Abstract, Chemical Abstracts, Embase, Index Copernicus, EBSCO, African Index Medicus, JournalSeek, Journal Citation Reports/Science Edition, Directory of Open Access Journals (DOAJ), African Journal Online, Bioline International, Open-J-Gate and Pharmacy Abstracts

\section{INTRODUCTION}

Primary lung carcinoma is one of the leading cause of cancer death in the world, with the number of diagnosed cases growing rapidly. [1]. Non-small cell lung cancer (NSCLC) is a major histological subtype of lung cancer, accounting for $80 \%$ of primary lung carcinoma cases [2]. Surgery, chemotherapy and radiotherapy are the main therapeutic strategies for managing primary lung carcinoma [3]. To improve the clinical outcomes in NSCLC patients, novel molecular targeted therapy has been utilized [4]. Individualized therapy is conducted in order to prolong the survival chances of advanced NSCLC patients [5]. Nevertheless, sensitive biomarkers which support individualized therapy for primary lung carcinoma are limited. Circular RNAs (CircRNAs) are a type of unique noncoding RNAs and they have been highlighted 
in recent research. They have a closed loop structure, and are resistant to RNA exonucleases [6]. CircRNAs are enriched by miRNA binding sites, which serve miRNA sponge effect as ceRNAs. In recent times, research has shown the close relationship between abnormally expressed circRNAs and cancer. CircRNAs exert vital regulations on malignant phenotypes of cancer cells and cancer metabolism. It has been reported that circLONP2 triggers invasiveness and metastasis of colorectal carcinoma by regulating the maturation and secretion of miRNAs [7]. Circ_0032821 serves as an oncogene that mediates growth, metastasis and autophagy of gastric cancer cells via the MEK1/ERK1/2 signaling [8].

Malignant gastric cancer can be alleviated by circMRPS35-induced histone modification on KAT7 [9]. Previous studies have demonstrated the oncogenic role of circZNF652 in renal carcinoma and hepatocellular carcinoma through the targeting of EMT and corresponding miRNAs [10]. In this study, circZNF652 demonstrated abnormal expression in primary lung carcinoma cases. Its potential influence on the malignant process of primary lung carcinoma and the underlying mechanism were explored in detail.

\section{METHODS}

\section{Subjects and samples}

Twenty-three primary lung carcinoma patients who were admitted in our hospital from May 2012 to December 2013 year were recruited for retrospective analysis. They did not have preoperative chemotherapy or radiotherapy. Cancer and paracancerous tissues that were 3 $\mathrm{cm}$ away from cancerous lesions were sampled and pathologically confirmed. Recruited patients were followed up for 5 years, up till December 2018. Tumor, Node, Metastasis (TNM) staging of primary lung carcinoma was as defined by UICC criteria. This study was approved by the research ethics committee of Taizhou Hospital of traditional Chinese Medicine (approval no. JS652-TZ-02-193). Informed consent was obtained from the patients. This study was conducted in accordance with the guidelines of the Declaration of Helsinki [11].

\section{Quantitative real-time polymerase chain reaction (qRT-PCR)}

Qualified RNA isolated from cells or tissues was reversely transcribed into cDNA using the PrimeScript RT reagent Kit (TaKaRa, Dalian, China). The cDNA was subjected to qRT-PCR using the SYBR Green Master Mix (Applied
Biosystems, San Diego, CA, USA). Each sample was made in triplicate, and the relative level was calculated by $2^{-\Delta \Delta \mathrm{Ct}}$. The primers used are shown in Table 1.

Table 1: Primer sequences used

\begin{tabular}{lll}
\hline Gene & & Primer sequence \\
\hline Circ- & Forward & 5'-GGGCACAAACAGTTCATGTG-3' \\
ZNF652 & Reverse & 5'-TGCGTTTGAATGATTTTCCA-3' \\
MiR-766 & Forward & 5'-TCGAGT \\
& & ACTTGAGATGGAGTTTT-3' \\
& Reverse & 5'- \\
& GGCCGCGTTGCAGTGAGCCGAG-3' \\
U6 & Forward & 5'-CTCGCTTCGGGCAGCACA-3' \\
& Reverse & 5'-AACGCTTCACGAAT TTGCGT-3' \\
GAPDH & Forward & 5'-AATGGACAACT GGTCGTGGAC-3' \\
& Reverse & 5'-CCCTCCAGGGGATCTGTTTG-3' \\
\hline
\end{tabular}

\section{Cell culture}

Lung carcinoma cell lines (NCl-H292, A549, SPC-A-1 and $\mathrm{NCl}-\mathrm{H} 460)$ and bronchial epithelial cell line $(16 \mathrm{HBE})$ were provided by Shanghai Institute of Biochemistry and Cell Biology (Shanghai, China). The cells were cultured in Roswell Park Memorial Institute 1640 (RPMI 1640) containing $10 \%$ fetal bovine serum (FBS) (Gibco, Rockville, MD, USA) and $1 \%$ penicillinstreptomycin.

\section{Transfection}

The CircZNF652 siRNA (si-circZNF652) and siNC were purchased from Invitrogen, Carlsbad, CA, USA. The MiR-766 mimics and inhibitor, as well as negative controls were synthesized by RiboBio, Guangzhou, China. Transfection was conducted using Lipofectamine 2000 (Invitrogen, Carlsbad, CA, USA).

\section{Cell Counting Kit (CCK-8) assay}

The cells were inoculated in a 96-well plate at a density of $2 \times 10^{3}$ cells/well. At $0,24,48$ and 72 $\mathrm{h}$, optical density at $450 \mathrm{~nm}$ of each sample was recorded using the CCK-8 kit (Dojindo Laboratories, Kumamoto, Japan) for plotting the viability curves.

\section{Transwell assay}

$5 \times 10^{4}$ cells were implanted on the top chamber, while $500 \mu \mathrm{l}$ of medium was applied on the bottom of a Transwell chamber. One hour later, migratory cells on the bottom were reacted with 15-min methanol and 20-min crystal violet, and then captured using a microscope. Migratory cells were counted in 10 randomly selected fields in each sample.

Trop J Pharm Res, November 2021; 20(11): 2256 


\section{Wound healing assay}

Cells were prepared into suspension with $1 \times 10^{5}$ cells $/ \mathrm{ml}$, and implanted in 6 -well plates. When 80 $\%$ of the cells had attached, an artificial scratch was made using a sterilized pipette tip. The cells were washed in phosphate buffered saline (PBS) 2-3 times and cultured in the medium containing $1 \%$ FBS. 24 hours later, the percentage of wound healing was calculated based on the migrated distance.

\section{Dual-luciferase reporter assay}

Sequences containing wild-type or mutant-type binding sites between circZNF652 and miR-766 were inserted in pmiR-RB-REPORT for synthesizing circZNF652-WT and circZNF652MUT, respectively. They were co-transfected in cells with miR-766 NC or miR-766 mimics for 48 $h$, followed by measure of assay luciferase activity.

\section{Western blot}

Cellular proteins were isolated using RIPA lysis buffer (Beyotime, Haimen, China), which were separated by sodium dodecyl sulphatepolyacrylamide gel electrophoresis (SDS-PAGE) and loaded on polyvinylidene fluoride (PVDF) membranes (Millipore, Billerica, MA, USA). After incubation in $5 \%$ skim milk for $2 \mathrm{~h}$, membranes were washed and cultured with primary and secondary antibodies. Band exposure was conducted using electrochemiluminescence (ECL) (Pierce, Rockford, IL, USA).

\section{Statistical analysis}

Data are expressed as mean \pm standard deviation, and processed using GraphPad Prism 6.0 (La Jolla, CA, USA). Differences between two groups were analyzed using the Student's t-test. Comparison between multiple groups was done using One-way ANOVA followed by Post-hoc test (Least Significant Difference). Kaplan-Meier curves were plotted for survival analysis. $P<$ 0.05 was considered indicative of statistically significant difference.

\section{RESULTS}

\section{CircZNF652 was upregulated in primary lung carcinoma cases}

Compared with normal tissues, circZNF652 was upregulated in primary lung carcinoma ones (Figure 1 A). As expected, circZNF652 was highly expressed in lung carcinoma cell lines (Figure $1 \mathrm{~B}$ ). According to the follow-up data of recruited patients, Kaplan-Meier curves presented a poor prognosis in primary lung carcinoma patients expressing high level of circZNF652, and showing a certain prognostic value (Figure 1C).

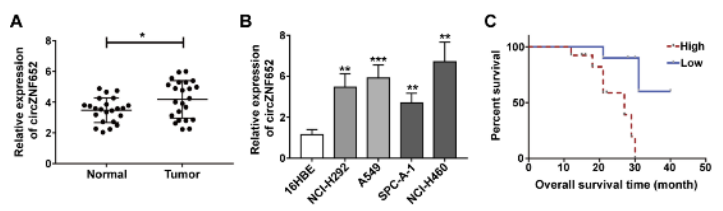

Figure 1: CircZNF652 was upregulated in primary lung carcinoma cases. (A) CircZNF52 levels in primary lung carcinoma as well as normal tissues; (B) CircZNF52 levels in lung carcinoma cell lines; (C) Overall survival in primary lung carcinoma patients expressing high or low level of circZNF652. ${ }^{*} P<0.01$

\section{CircZNF652 stimulated proliferative and migratory capacities in primary lung carcinoma}

i-circZNF652 was synthesized and transfected in A549 and SPC-A-1 cells for silencing circZNF652 (Figure $2 \mathrm{~A}$ ). The knockdown of circZNF652 remarkably reduced viability in A549 and SPC-A1 cells, indicating the inhibited proliferative capacity (Figure $2 \mathrm{~B}$ and $\mathrm{C}$ ). Moreover, wound healing assay and Transwell assay yielded the conclusion that the knockdown of circZNF652 suppressed migratory capacity in primary lung carcinoma (Figure 2 D and E). In addition, protein levels of $\mathrm{N}$-cadherin and Vimentin were downregulated, whereas E-cadherin was upregulated in A549 and SPC-A-1 cells transfected with si-circZNF652 (Figure $2 \mathrm{~F}$ and $\mathrm{G})$. This indicated that circZNF652 was able to affect EMT in primary lung carcinoma, which may be a key mechanism for the oncogenic role of circZNF652.

\section{CircZNF652 binding negatively regulated miR-766}

To elucidate on how circZNF652 exerts oncogenic role in primary lung carcinoma, its potential target was predicted using online software. Binding sites were identified in the 3'UTR of circZNF652 and miR-766 (Figure 3 A). Subsequently, dual-luciferase reporter assay confirmed the binding relationship between circZNF652 and miR-766 (Figure $3 \mathrm{~B}$ and C). Transfection of si-circZNF652 markedly upregulated miR-766 level in A549 and SPC-A-1 cells (Figure 3 D). Consistently, a negative correlation was identified between circZNF652 and miR-766 in primary lung carcinoma tissues (Figure $3 \mathrm{E}$ ).

Trop J Pharm Res, November 2021; 20(11): 2257 


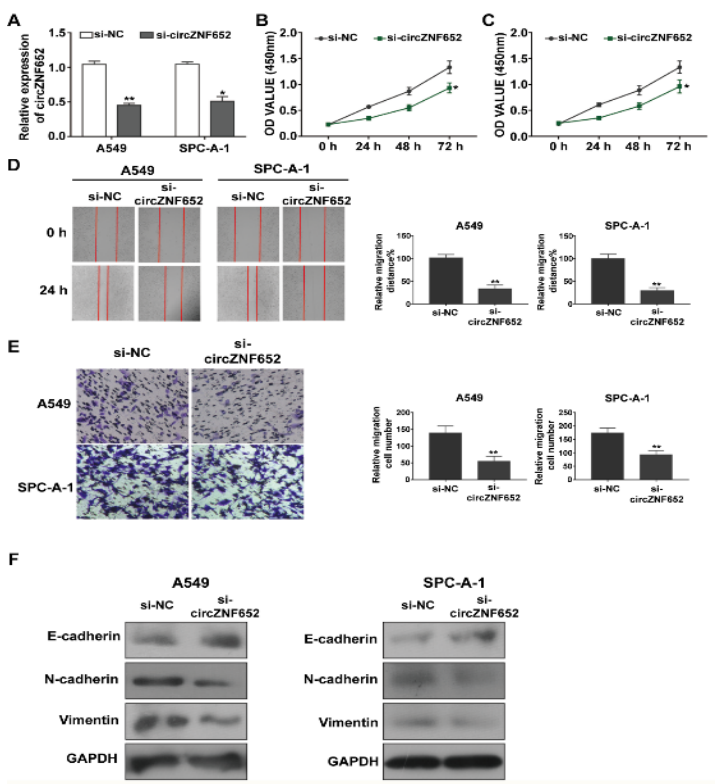

Figure 2: CircZNF652 stimulated proliferative and migratory capacities in primary lung carcinoma. (A) Transfection efficacy of si-circZNF652 in A549 and SPC-A-1 cells; (B, C) Viability of A549 and SPC-A-1 cells with circZNF652 knockdown; (D) Wound healing ability in A549 and SPC-A-1 cells with circZNF652 knockdown; (E) Migration capacities of A549 and SPC-A-1 cells with circZNF652 knockdown; $(F, G)$ Protein levels of E-cadherin, $\mathrm{N}$-cadherin and Vimentin in A549 and SPC-A-1 cells with circZNF652 knockdown. ${ }^{\star \star} P<0.01,{ }^{* \star *} p<0.001$

A
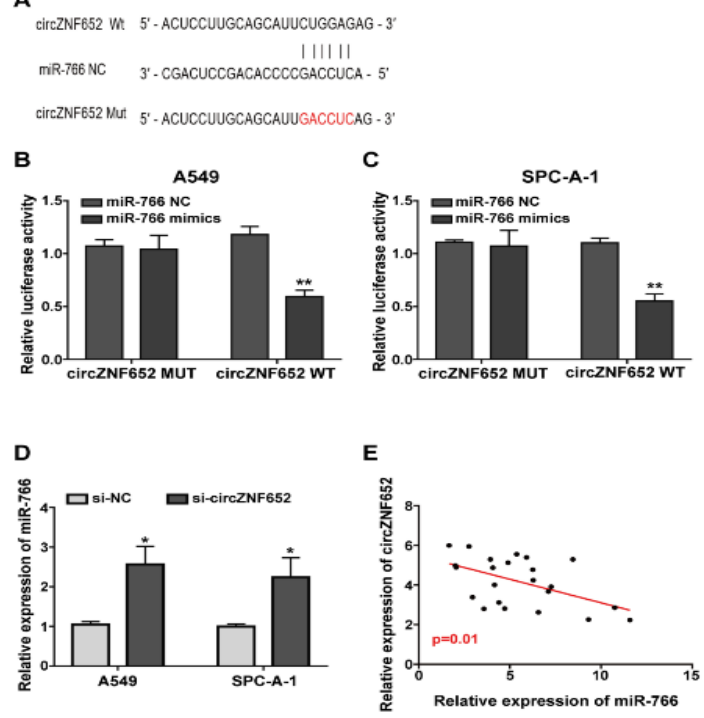

Figure 3: CircZNF652 bound to negatively regulated miR-766. (A) Binding sites in the 3'UTR of circZNF652 and miR-766; $(B, C)$ binding relationship between circZNF652 and miR-766; (D) MiR-766 levels in A549 and SPC-A-1 cells with circZNF652 knockdown; (E) negative correlation between circZNF652 and miR-766 in primary lung carcinoma tissues. ${ }^{* *} P<0.01$
MiR-766 was the key downstream gene for the oncogenic role of circZNF652

Focus was thereafter shifted to investigating the involvement of miR-766 in primary lung carcinoma. CCK-8 assay showed a higher viability in A549 and SPC-A-1 cells, with cosilence of circZNF652 and miR-766, than in those with knockdown of circZNF652 (Figure $4 \mathrm{~A}$ and B). Besides, knockdown of miR-766 partially reversed the declined migratory cell number and percentage of wound healing closure of lung carcinoma cells with circZNF652 knockdown (Figure $4 \mathrm{C}-\mathrm{F}$ ). Taken together, knockdown of miR-766 abolished the role of circZNF652 in regulating primary lung carcinoma phenotypes.
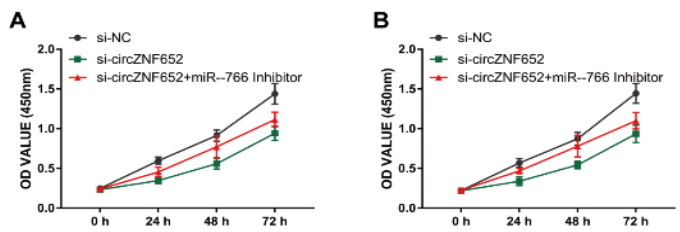

C

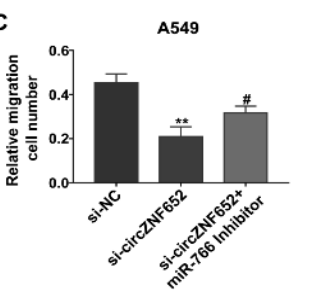

D
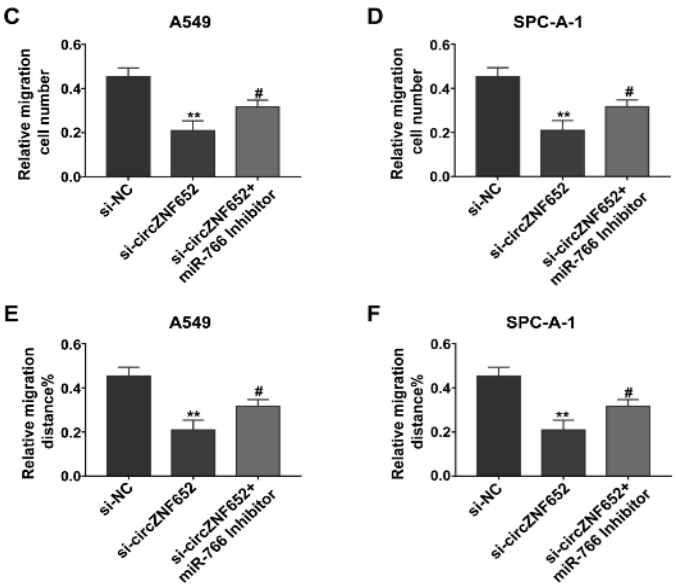

Figure 4: MiR-766 was the key downstream gene for the oncogenic role of circZNF652. (A, B) Viability in A549 and SPC-A-1 cells with co-knockdown of circZNF652 and miR-766; (C, D) wound healing ability of A549 and SPC-A-1 cells with co-knockdown of circZNF652 and miR-766; (E, F) migration of A549 and SPC-A-1 cells with co-knockdown of circZNF652 and miR-766. ${ }^{* *} P<0.01$

\section{DISCUSSION}

Lung carcinoma is a highly lethal cancer of the respiratory system, and its casualty numbers rank amongst the highest when it comes to malignancies [12]. For a long period of time, anticancer treatments for primary lung carcinoma were limited, and a variety of effective strategies have now been applied in clinical diagnosis and treatment, including immunotherapy and targeted therapy. The pathogenesis of lung carcinoma is complicated, and it includes EMT, disorder of 
intracellular environment, and reprogramming of cell metabolism [13]. With the process of genomics, genetic heterogeneity has been identified as vital in carcinogenesis, including abnormally activated tumor-associated genes and epigenetic modification [14]. Recently, circRNAs are considered as novel biomarker for cancers because of their high conservatism, stability and specificity. They contribute to form a regulatory network involved in the regulation of cancer epigenetic modification $[15,16]$.

Previous findings have demonstrated that circZNF652 is a vital oncogene $[17,18]$. Consistently, this study showed that circZNF652 was upregulated in primary lung carcinoma cases and highly expressed circZNF652 was unfavorable to the prognosis of the patients. A series of functional experiments verified that circZNF652 stimulated proliferative and migratory capacities, as well as EMT in A549 and SPC-A-1 cells.

To further clarify the molecular mechanism of circZNF652 in regulating primary lung carcinoma, its molecular target was determined using an online software (https://circinteractome.nia.nih. gov/), and miR-766 was selected. The MiR-766 is confirmed as a tumor-suppressor gene, and it was reported that it alleviates the process of hepatocellular carcinoma by directly targeting the Wnt signaling [19].

MiR-766 prevents the clinical progression of colorectal carcinoma by limiting cancer cell metastasis [20]. Besides, through the activation of the well-known p53, miR-766 participated in cell cycle progression of cancer cells [21]. In lung adenocarcinoma and NSCLC, miR-766 is a potential regulator that influences cancer progression [22,23]. It induces the biological function of noncoding RNAs and thus determines lung carcinoma aggravation [24]. In this study, miR-766 was the downstream gene of circZNF652, presenting a negative correlation between each other. Notably also, the knockdown of miR-766 was able to abolish the inhibited proliferative and migratory capacities in A549 and SPC-A-1 cells with circZNF652 knockdown. We have demonstrated a negative axis circZNF652/miR-766 that was responsible for stimulating the malignant process of primary lung carcinoma.

\section{CONCLUSION}

The results obtained indicate that CircZNF652 serves as an oncogene that triggers the aggravation of primary lung carcinoma by negatively regulating miR-766. Thus,
CircZNF652 is a promising target for the treatment of primary lung carcinoma.

\section{DECLARATIONS}

\section{Conflict of Interest}

No conflict of interest associated with this work.

\section{Contribution of Authors}

The authors declare that this work was done by the authors named in this article and all liabilities pertaining to claims relating to the content of this article will be borne by them.

\section{Open Access}

This is an Open Access article that uses a funding model which does not charge readers or their institutions for access and distributed under the terms of the Creative Commons Attribution License (http://creativecommons.org/licenses/by/ 4.0) and the Budapest Open Access Initiative (http://www.budapestopenaccessinitiative.org/rea d), which permit unrestricted use, distribution, and reproduction in any medium, provided the original work is properly credited.

\section{REFERENCES}

1. Miller $K D$, Nogueira $L$, Mariotto $A B$, Rowland $J H$, Yabroff $K R$, Alfano CM, Jemal A, Kramer JL, Siegel RL. Cancer treatment and survivorship statistics, 2019. CA Cancer J Clin 2019; 69(5): 363-385.

2. M TM, O RE, M PA, K IM, V MS, V CO, G FI, A TS, VLN. Prognostic significance of ERCC1, RRM1, TOP1, TOP2A, TYMS, TUBB3, GSTP1 AND BRCA1 mRNA expressions in patients with non-small-cell lung cancer receiving a platinum-based chemotherapy. J Buon 2020; 25(4): 1728-1736.

3. Jankovic R, J GH, Cavic M, Clemente C, Lind M, Murillo $C A$, Nadifi S, Khyatti M, Adebambo T, Egamberdiev $D$. LungCARD - Report on worldwide research and clinical practices related to lung cancer. J Buon 2019; 24(1): 1119.

4. Mayekar MK, Bivona TG. Current Landscape of Targeted Therapy in Lung Cancer. Clin Pharmacol Ther 2017; 102(5): 757-764.

5. Mott TF. Lung Cancer: Management. FP Essent 2018; 464: 27-30.

6. Yang B, Zhao J, Huo $T$, Zhang $M$, Wu X. Effects of CirCRNA-ITCH on proliferation and apoptosis of hepatocellular carcinoma cells through inhibiting Wnt/beta-catenin signaling pathway. J Buon 2020; 25(3): 1368-1374.

7. Han K, Wang FW, Cao CH, Ling H, Chen JW, Chen RX, Feng $Z H$, Luo J, Jin XH, Duan JL, et al. CircLONP2

Trop J Pharm Res, November 2021; 20(11): 2259 
enhances colorectal carcinoma invasion and metastasis through modulating the maturation and exosomal dissemination of microRNA-17. Mol Cancer 2020; 19(1): 60.

8. Jiang $Y$, Zhang $Y$, Chu F, Xu L, Wu H. Circ_0032821 acts as an oncogene in cell proliferation, metastasis and autophagy in human gastric cancer cells in vitro and in vivo through activating MEK1/ERK1/2 signaling pathway. Cancer Cell Int 2020; 20: 74.

9. Jie $M, W u Y$, Gao $M$, Li $X$, Liu C, Ouyang $Q$, Tang $Q$, Shan $C, L V Y$, Zhang $K$, et al. CircMRPS35 suppresses gastric cancer progression via recruiting KAT7 to govern histone modification. Mol Cancer 2020; 19(1): 56.

10. Zhang L, Guo Y. Silencing circular RNA-ZNF652 represses proliferation and EMT process of renal carcinoma cells via raising miR-205. Artif Cells Nanomed Biotechnol 2020; 48(1): 648-655.

11. Stockhausen $K$. The Declaration of Helsinki: revising ethical research guidelines for the 21st century. Med J Aust 2000; 172(6): 252-253.

12. Hirsch FR, Scagliotti GV, Mulshine JL, Kwon R, Curran WJ, Wu YL, Paz-Ares L. Lung cancer: current therapies and new targeted treatments. Lancet 2017; 389(10066): 299-311.

13. Gao Y, Chen J, Zhang J, Sun L, Zhuang Y. Radiofrequency ablation of primary non-small cell lung cancer: A retrospective study on 108 patients. J Buon 2019; 24(4): 1610-1618.

14. Miron O, Afrasanie VA, Paduraru MI, Trandafir LM, Miron $L$. The relationship between chronic lung diseases and lung cancer - a narrative review. J Buon 2020; 25(4): 1687-1692.

15. Hu W, Bi ZY, Chen ZL, Liu C, Li LL, Zhang F, Zhou Q, Zhu $W$, Song $Y Y$, Zhan BT, et al. Emerging landscape of circular RNAs in lung cancer. Cancer Lett 2018; 427: 18-27.

16. Beermann J, Piccoli MT, Viereck J, Thum T. Non-coding RNAs in Development and Disease: Background,
Mechanisms, and Therapeutic Approaches. Physiol Rev 2016; 96(4): 1297-1325.

17. Zhang L, Guo Y. Silencing circular RNA-ZNF652 represses proliferation and EMT process of renal carcinoma cells via raising miR-205. Artif Cells Nanomed Biotechnol 2020; 48(1): 648-655.

18. Guo J, Duan H, Li Y, Yang L, Yuan L. A novel circular RNA circ-ZNF652 promotes hepatocellular carcinoma metastasis through inducing snail-mediated epithelialmesenchymal transition by sponging miR-203/miR-5025p. Biochem Biophys Res Commun 2019; 513(4): 812819.

19. Liu L, Qi X, Gui $Y$, Huo $H$, Yang $X$, Yang $L$. Overexpression of circ_0021093 circular RNA forecasts an unfavorable prognosis and facilitates cell progression by targeting the miR-766-3p/MTA3 pathway in hepatocellular carcinoma. Gene 2019; 714: 143992.

20. Jia B, Xia L, Cao F. The role of miR-766-5p in cell migration and invasion in colorectal cancer. Exp Ther Med 2018; 15(3): 2569-2574.

21. Wang Q, Selth LA, Callen DF. MiR-766 induces p53 accumulation and G2/M arrest by directly targeting MDM4. Oncotarget 2017; 8(18): 29914-29924.

22. Wang $M$, Liao $Q$, Zou P. PRKCZ-AS1 promotes the tumorigenesis of lung adenocarcinoma via sponging miR-766-5p to modulate MAPK1. Cancer Biol Ther 2020; 21(4): 364-371.

23. Cai $Y$, Jia $R$, Xiong $H$, Ren $Q$, Zuo $W$, Lin $T$, Lin $R$, Lei $Y$, Wang $P$, Dong $H$, et al. Integrative gene expression profiling reveals that dysregulated triple microRNAs confer paclitaxel resistance in non-small cell lung cancer via co-targeting MAPT. Cancer Manag Res 2019; 11: 7391-7404.

24. Bai Y, Zhang G, Cheng R, Yang R, Chu H. CASC15 contributes to proliferation and invasion through regulating miR-766-5p/ KLK12 axis in lung cancer. Cell Cycle 2019; 18(18): 2323-2331. 